\title{
Aberrant expression of miR-130a-3p in ankylosing spondylitis and its role in regulating T-cell survival
}

\author{
FENGJU LI ${ }^{1}$, DINGRAN $\mathrm{SI}^{2}$, XUEJUN GUO ${ }^{3}$, NINGRU GUO $^{1}$, DANDAN LI ${ }^{1}$, \\ LIUJING ZHANG $^{1}$, XIANAN JIAN ${ }^{1}$ and JIASHENG MA ${ }^{1}$ \\ Departments of ${ }^{1}$ Rheumatism and Immunology, ${ }^{2}$ Cardiovascular Medicine and ${ }^{3}$ Hematology, \\ Puyang Oilfield General Hospital, Puyang, Henan 457001, P.R. China
}

Received August 7, 2018; Accepted March 6, 2019

DOI: $10.3892 / \mathrm{mmr} .2019 .10573$

\begin{abstract}
Ankylosing spondylitis (AS) is a chronic inflammatory rheumatic disease. MicroRNAs (miRNAs) are a group of endogenous small non-coding RNAs that regulate target genes, and play a critical role in many biological processes. However, the underlying mechanism of specific miRNA, miR-130a-3p, in AS remains largely unknown. Therefore, the present study aimed to explore the underlying mechanism of miR-130a-3p in the development of AS. In the present study, it was revealed that miR-130a-3p was downregulated in $\mathrm{T}$ cells from HLA-B27-positive AS patients compared with the HLA-B27-negative healthy controls. Next, bioinformatics software TargetScan 7.2 was used to predict the target genes of miR-130a-3p, and a luciferase reporter assay indicated that HOXB1 was the direct target gene of miR-130a-3p. Furthermore, it was determined that HOXB1 expression was upregulated in T cells from HLA-B27-positive AS patients. In addition, the results of the present study indicated that miR-130a-3p inhibitor significantly inhibited cell proliferation ability and induced cell apoptosis of Jurkat $\mathrm{T}$ cells, while the miR-130a-3p mimic promoted proliferation ability and inhibited cell apoptosis of Jurkat T cells. Notably, all the effects of the miR-130a-3p mimic on Jurkat $\mathrm{T}$ cells were reversed by HOXB1-plasmid. Collectively, our data indicated that miR-130a-3p was decreased in T cells from AS patients and it could regulate T-cell survival by targeting HOXB1.
\end{abstract}

\section{Introduction}

Ankylosing spondylitis (AS) is a chronic inflammatory rheumatic disease that is characterized by pain and stiffness between the spinal bones. AS mainly affects the axial skeleton, leading to back pain, progressive structural and functional

Correspondence to: Dr Dingran Si, Department of Cardiovascular Medicine, Puyang Oilfield General Hospital, 124 Daqing Road, Puyang, Henan 457001, P.R. China

E-mail: sidr201807@163.com

Key words: ankylosing spondylitis, miR-130a-3p, HOXB1, T cells impairment and reduced quality of life (1). It has been recognized that HLA-B27 is the most important risk factor for AS (2). The prevalence of AS is $\sim 0.20-0.40 \%$ in China (3-5). The patients are predominantly young adults, with more male patients than female patients, and the disability rate 5 years after the onset of symptoms is 40-60\% (3,4,6,7). Genome-wide association studies (GWAS) have investigated many genes related with $\mathrm{AS}$, however, to date, the pathogenesis of AS has not been well elucidated. Recently, it was reported that aberrant apoptosis of $\mathrm{T}$ cells was observed in peripheral blood and inflammatory joints of AS patients.

MicroRNAs (miRNAs) are a large family of highly conserved endogenous small non-coding RNAs that are 21-23 nucleotides in length in eukaryotes (8). miRNAs do not encode proteins, and they can inhibit the expression of multiple target genes by binding to the 3 prime-untranslated region (3'-UTR) of target mRNAs (9-11). In addition, miRNAs post-transcriptionally regulate the translation and degradation of their target genes. miRNAs are associated with inflammatory response and the development of autoimmune diseases, for example, rheumatoid arthritis (12), and multiple sclerosis (13). In recent years, research on miRNAs in AS has received more attention. Lai et al reported the first research on miRNA expression profiling in AS from 5 AS patients and 5 healthy controls (14). Their study revealed that the expression of 8 miRNAs such as miR-150, miR-342-5p, miR-16, miR-221, miR-99b, let-7b, let-7i, and miR-513-5p were upregulated and 5 miRNAs such as miR-218, miR-30e, miR-199a-5p, miR-409-3p and miR-215 were downregulated in the T cells of AS compared with their normal counterparts. A recent study demonstrated that deacetylase (HDAC) 3-mediated suppression of miR-130a in peripheral blood mononuclear cells (PBMCs) from AS patients was involved in increased expression of its target tumor necrosis factor (TNF)- $\alpha$ (15). Moreover, that study revealed that miR-130a was significantly downregulated in immune cells in AS patients. These results indicated that miR-130a-3p may play an important role in the development of AS. However, the function and downstream molecular pathways of miR-130a-3p during the pathogenesis of AS are unclear.

The HOX gene encodes a family of highly conserved transcription factors. The gene contains a helix-turn-helix DNA binding domain that has 60 amino acids, and it plays a crucial 
role in growth and development, regulating many biological processes including apoptosis, receptor signaling, differentiation, movement and angiogenesis (16). It has been reported that HOXB1, as a tumor suppressor gene, could be regulated by miR-3175 in glioma (17). However, the role of HOXB1 in AS remains unclear. In the present study, through bioinformatics software analysis the binding site between miR-130a-3p and HOXB1 was revealed. Therefore, we surmised that miR-130a-3p may play a role in regulating T-cell survival by regulating $\mathrm{HOXB1}$.

In the present study, we aimed to confirm the expression of miR-130a-3p in T cells of AS patients and to further explore its effects on $\mathrm{T}$ cells, to elucidate whether miR-130a-3p participates in the development of AS by regulating T-cell survival. These findings may provide insight for the development of new approaches to manage AS.

\section{Materials and methods}

Study population. In total, 30 paired blood samples were collected from 30 HLA-B27-positive AS patients (female to male ratio, 1:4; age range, 22-61 years) and 30 HLA-B27-negative healthy subjects (female to male ratio, $1: 4$; age range, $19-58$ years) who had no history of autoimmune diseases in our hospital between December 2015 and February 2018 . Blood samples were stored at $-80^{\circ} \mathrm{C}$ until use. The present study was approved by the Ethics Committee of Puyang Oilfield General Hospital (Puyang, China), and written informed consent was obtained from every patient.

T-cell isolation and culture. Venous blood from AS patients and healthy volunteers was collected into tubes containing EDTA, from which PBMCs were isolated by Ficoll-Hypaque density gradient centrifugation at $300 \mathrm{x}$ g and $4^{\circ} \mathrm{C}$ for $10 \mathrm{~min}$. $\mathrm{T}$ cells were further purified using magnetic beads coated with anti-human CD33 (Miltenyi Biotec GmbH, Gladbach, Germany). The T cells were cultured in RPMI-1640 medium (Gibco; Thermo Fisher Scientific, Inc., Waltham, MA, USA) supplemented with $10 \%$ FBS (Gibco; Thermo Fisher Scientific, Inc.), $2 \mathrm{mM}$ L-glutamine (Beyotime Institute of Biotechnology, Haimen, China), $100 \mathrm{U} / \mathrm{ml}$ penicillin (Beyotime Institute of Biotechnology), and $100 \mathrm{U} / \mathrm{ml}$ streptomycin (Beyotime Institute of Biotechnology) at $37^{\circ} \mathrm{C}, 5 \% \mathrm{CO}_{2}$ incubator.

Cell culture and cell transfection. The human Jurkat cells were purchased from Shanghai Institute of Life Sciences, Chinese Academy of Sciences (Shanghai, China) and cultured in Dulbecco's modified Eagle's medium (DMEM) supplemented with $10 \% \mathrm{FBS}$, and incubated at $37^{\circ} \mathrm{C}$, in $5 \% \mathrm{CO}_{2}$ incubator. Jurkat T cells were transfected with $50 \mathrm{nM}$ inhibitor control (5'-CAGUACUUUUGUGUAGUACAA-3'), $50 \mathrm{nM}$ miR-130a-3p inhibitor (5'-AUGCCCUUUUAACAUUGC ACUG-3), $50 \mathrm{nM}$ mimic control (5'-UUCUCCGAACGU GUCACGUTT-3'), 50 nM miR-130a-3p mimic (5'-CAGUGC AAUGUUAAAAGGGCAU-3'; all from Guangzhou RiboBio Co., Ltd., Guangzhou, China), $10 \mu \mathrm{M}$ control-plasmid, $10 \mu \mathrm{M}$ HOXB1-plasmid, or miR-130a-3p mimic+HOXB1-plasmid using Lipofectamine 2000 reagent (Invitrogen; Thermo Fisher Scientific, Inc.) following the manufacturer's instructions. Cell transfection efficiency was detected $48 \mathrm{~h}$ after transfection.
$R T$ - $q P C R$. Total RNA from blood/cells was extracted using TRIzol reagent (Invitrogen; Thermo Fisher Scientific, Inc.) according to the manufacturer's instructions. The RNA concentration was detected by NanoDrop 2000. RT-qPCR was carried out using 2X SYBR-Green PCR Master Mix (Applied Biosystems; Thermo Fisher Scientific, Inc.). Reaction conditions were as follows: $10 \mathrm{~min}$ at $95^{\circ} \mathrm{C}$ followed by 35 cycles of $15 \mathrm{sec}$ at $95^{\circ} \mathrm{C}$ and $40 \mathrm{sec}$ at $55^{\circ} \mathrm{C}$. The primer sequences used for RT-qPCR are listed in Table I. The relative gene expression levels were calculated using the $2^{-\Delta \Delta \mathrm{Cq}}$ method (18) after normalization with reference the expression of GAPDH or U6. All experiments were performed in triplicate.

Western blot analysis. Total proteins were extracted from cells using radioimmunoprecipitation assay buffer (Beyotime Institute of Biotechnology), according to the manufacturer's protocol. A bicinchoninic acid protein assay kit (Thermo Fisher Scientific, Inc.) was used to analyze the protein concentration. The protein samples (35 $\mu \mathrm{g} /$ lane) were separated on $10 \%$ sodium dodecyl sulfate-polyacrylamide gel electrophoresis (SDS-PAGE) gel and transferred to polyvinylidene fluoride (PVDF) membranes. The membranes were blocked with $5 \%$ non-fat milk for $1.5 \mathrm{~h}$, and were incubated overnight at $4^{\circ} \mathrm{C}$ with the following primary antibodies: $\beta$-actin $(1: 1,000$; cat. no. 4970; Cell Signaling Technology, Inc.), HOXB1 (1:1,000; cat no. ab168279; Abcam), Bcl-2 (1:1,000; cat. no. 4223; Cell Signaling Technology, Inc.) and Bax (1:1,000; cat. no. 5023; Cell Signaling Technology, Inc.). Subsequently, the membranes were incubated with the anti-rabbit immunoglobulin $\mathrm{G}$ horseradish peroxidase-conjugated secondary antibody (1:5,000; cat no. 7074; Cell Signaling Technology, Inc.) for $2 \mathrm{~h}$ at room temperature. Proteins were detected using an ECL kit (Thermo Fisher Scientific, Inc.) and imaged. $\beta$-actin was used as an internal control, and the AlphaView 3.4.0 (ProteinSimple, San Jose, CA, USA) software was used for quantification analysis.

CCK-8 assay. Cell Counting Kit-8 (CCK-8) assay was performed to assess the cell proliferation ability $(16,19)$. Logarithmic phase cells were seeded in a 96-well plate with $1 \times 10^{4}$ cells/well and incubated at $37^{\circ} \mathrm{C}$ with $5 \% \mathrm{CO}_{2}$ for $12 \mathrm{~h}$, after which $10 \mu \mathrm{l} \mathrm{CCK}-8$ solution was added to each well, and the cells were incubated for a further $2 \mathrm{~h}$ at $37^{\circ} \mathrm{C}$ with $5 \% \mathrm{CO}_{2}$. The absorbance was measured at a wavelength of $450 \mathrm{~nm}$ using a microplate reader.

Flow cytometric assay. Jurkat T cells were transfected with inhibitor control, miR-130a-3p inhibitor, mimic control, miR-130a-3p mimic, or miR-130a-3p mimic+HOXB1-plasmid for $48 \mathrm{~h}$. Then, Jurkat $\mathrm{T}$ cells were collected at logarithmic growth phase, and Annexin V-fluorescein isothiocyanate (FITC)/propidium iodide (PI) apoptosis detection kit (cat. no. 70-AP101-100; MultiSciences Biotech Co., Ltd., Hangzhou, China) was used to analyze cell apoptosis. Briefly, Jurkat T cells were stained with $5 \mu \mathrm{l}$ Annexin V- FITC and $5 \mu \mathrm{PI}$ for $30 \mathrm{~min}$ at room temperature in the dark. A flow cytometer (BD Biosciences, Franklin Lakes, NJ, USA) was used to perform analysis of cell apoptosis.

Dual-luciferase reporter assay. Bioinformatics software TargetScan 7.2 (http://www.targetscan.org/vert_72/) was used to predict target genes of miR-130a-3p (20), and the results 
Table I. Primer sequences for PCR.

\begin{tabular}{ll}
\hline Gene & \multicolumn{1}{c}{ Sequence (5'-3') } \\
\hline Bcl-2 & F: 5'-TTGGATCAGGGAGTTGGAAG-3' \\
& R: 5'-TGTCCCTACCAACCAGAAGG-3' \\
Bax & F: 5'-CGTCCACCAAGAAGCTGAGCG-3' \\
& R: 5'-CGTCCACCAAGAAGCTGAGCG-3' \\
HOXB1 & F: 5'-CGTCCACCAAGAAGCTGAGCG-3' \\
& R: 5'-CGTCCACCAAGAAGCTGAGCG-3' \\
miR-130a-3p & F: 5'-GGCAGTGCAATGTTAAAAG-3' \\
& R: 5'-CAGTGCGTGTCGTGGAGT-3' \\
U6 & F: 5'-CTCGCTTCGGCAGCACA-3' \\
& R: 5'-AACGCTTCACGAATTTGCGT-3' \\
GAPDH & F: 5'-CTTTGGTATCGTGGAAGGACTC-3' \\
& R: 5'-GTAGAGGCAGGGATGATGTTCT-3'
\end{tabular}

F, forward; R, reverse.

revealed the binding sites between miR-130a-3p and HOXB1. To investigate the relationship between miR-130a-3p and HOXB1, a luciferase reporter vector that contained a 3'-UTR sequence of HOXB1 was constructed. Cells seeded in 24-well plates were co-transfected with miR-130a-3p mimic or mimic control and the mutant (MUT) or wild-type (WT) 3'-UTR of HOXB1 using Lipofectamine 2000 for 48 h, along with Renilla luciferase $\mathrm{pRL}-\mathrm{TK}$ vector as a control. After transfection for $48 \mathrm{~h}$, the cells were lysed with RIPA buffer. The relative luciferase activity was detected using a Dual-Luciferase Reporter Assay System (Promega Corporation, Madison, WI, USA) according to the manufacturer's instructions.

Statistical analyses. Each experiment was performed at least three times. Data analyses were performed using GraphPad Prism 6.0 (GraphPad Software, Inc., La Jolla, CA, USA). All data were expressed as the mean \pm standard deviation (SD). The significance of differences between groups was carried out by one-way analysis of variance with Tukey's post hoc test or Student's t-test. $\mathrm{P}<0.05$ was considered to indicate a statistically significant difference.

\section{Results}

Expression level of miR-130a-3p in T cells from AS patients. RT-qPCR was firstly performed to detect the level of miR-130a-3p in T cells in HLA-B27-positive AS patients and HLA-B27-negative healthy subjects. The results revealed that the expression level of miR-130a-3p was significantly downregulated in T cells in HLA-B27-positive AS patients compared with HLA-B27 negative healthy controls (Fig. 1).

HOXB1 is a direct target of miR-130a-3p. To identify the functional targets of miR-130a-3p, TargetScan was used. TargetScan revealed that miR-130a-3p has hundreds of target genes, including HOXB1 (Fig. 2A). HOXB1 regulates many biological processes including receptor signaling, differentiation, movement, angiogenesis, cell proliferation and apoptosis.

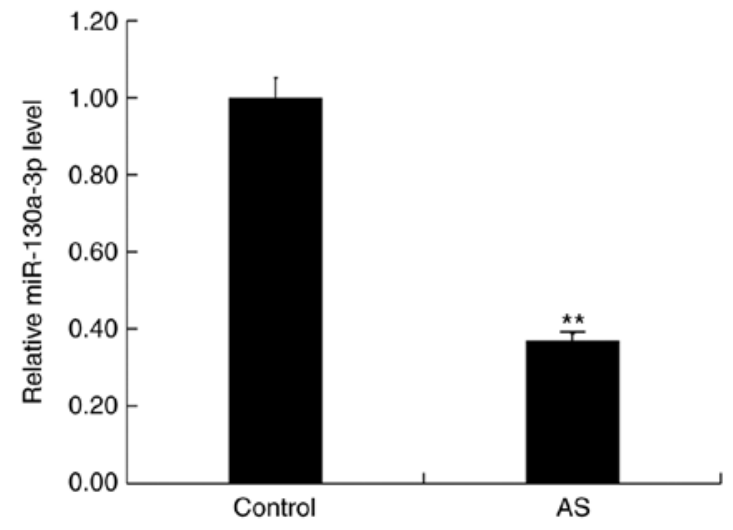

Figure 1. miR-130a-3p level in T cells in AS patients. The level of miR-130a-3p in T cells in HLA-B27-positive AS patients (AS) and HLA-B27 negative healthy controls (control) was detected using RT-qPCR. Data are displayed as the mean \pm SD. ${ }^{* *} \mathrm{P}<0.01$ vs. the control group. AS, ankylosing spondylitis; $\mathrm{SD}$, standard deviation.

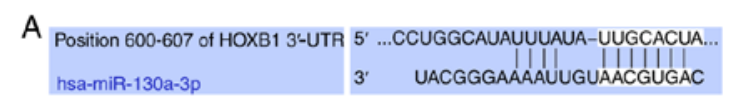

$\mathrm{B}$

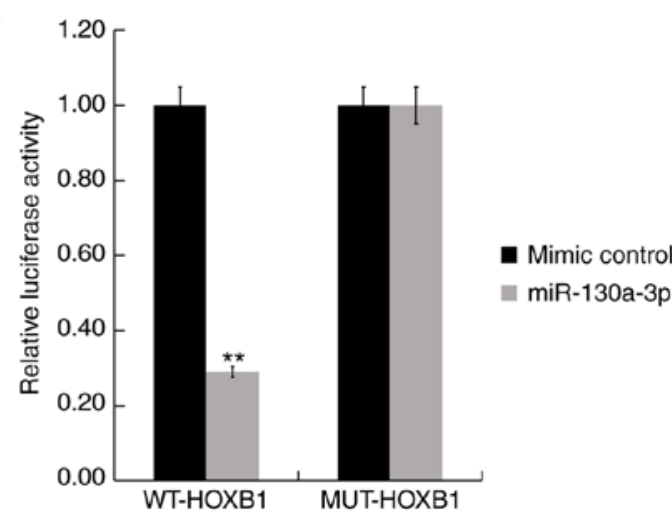

Figure 2. miR130a-3p directly suppresses HOXB1. (A) TargetScan predicted the HOXB1 3'-UTR binding site for miR-130a-3p. (B) A luciferase reporter assay was performed after Jurkat $\mathrm{T}$ cells were co-transfected with luciferase reporter plasmids containing HOXB1-3'-UTR-WT or HOXB1-3'-UTR-MUT, along with miR-130a-3p mimic or mimic control for $48 \mathrm{~h}$. Data are displayed as the mean $\pm \mathrm{SD}$. ${ }^{* *} \mathrm{P}<0.01$ vs. mimic control. 3'-UTR, 3 prime-untranslated region; WT, wild-type; MUT, mutant; SD, standard deviation.

However, the role of HOXB1 in AS remains largely unclear, therefore, HOXB1 was selected for further study. Furthermore, to further examine whether miR-130a-3p directly targets HOXB1, Luc HOXB1-3'-UTR-WT and its 3'-UTR MUT plasmids were constructed. A luciferase reporter assay revealed that miR-130a-3p mimic significantly suppressed the luciferase activity of the wild-type HOXB1 3'-UTR (Fig. 2B). These results provided evidence that $\mathrm{HOXB} 1$ was a direct target of miR-130a-3p.

Expression level of HOXB1 in T cells from AS patients. Next, the expression level of HOXB1 in T cells in HLA-B27-positive AS patients and HLA-B27 negative healthy subjects was detected by RT-qPCR and western blot assays. The results revealed that both the mRNA and protein levels of HOXB1 in T cells in HLA-B27-positive AS patients were higher than that in the HLA-B27 negative healthy subjects (Fig. 3). 

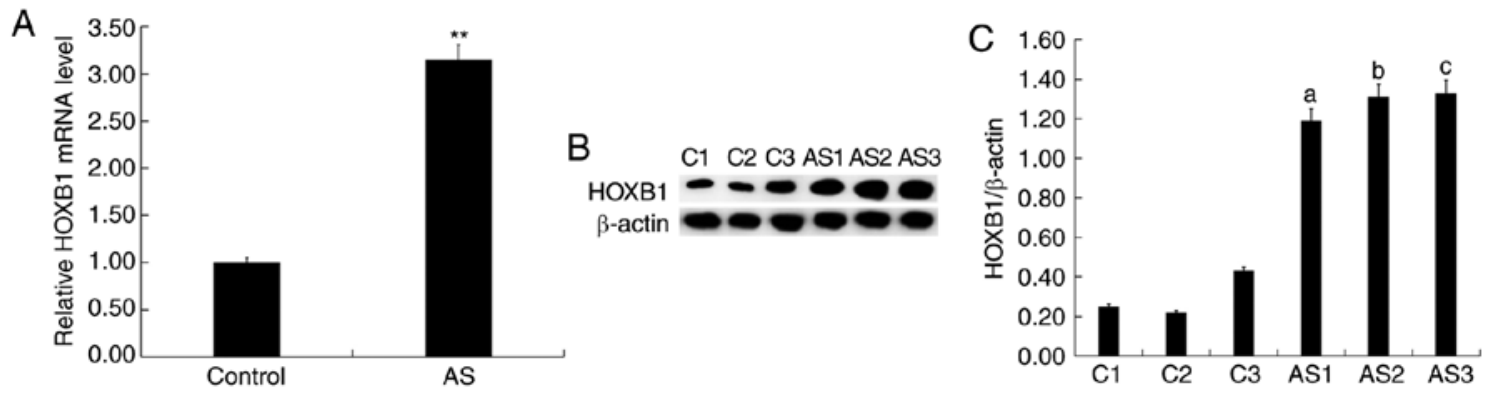

Figure 3. HOXB1 expression in T cells in AS patients. (A) RT-qPCR and (B) western blot analysis were used to assess the expression of HOXB1 in T cells in HLA-B27-positive AS patients (AS: AS1, AS2, AS3) and HLA-B27 negative healthy subjects (control: C1, C2, C3). (C) The HOXB1 protein level/ $\beta$-actin protein level was analyzed. Data are displayed as the mean $\pm \mathrm{SD}$. ${ }^{* *} \mathrm{P}<0.01$ vs. control group; ${ }^{\mathrm{a}} \mathrm{P}<0.05$ vs. $\mathrm{C} 1 ;{ }^{b} \mathrm{P}<0.05$ vs. $\mathrm{C} 2$; ${ }^{\mathrm{c}} \mathrm{P}<0.05$ vs. $\mathrm{C} 3$. AS, ankylosing spondylitis; SD, standard deviation.
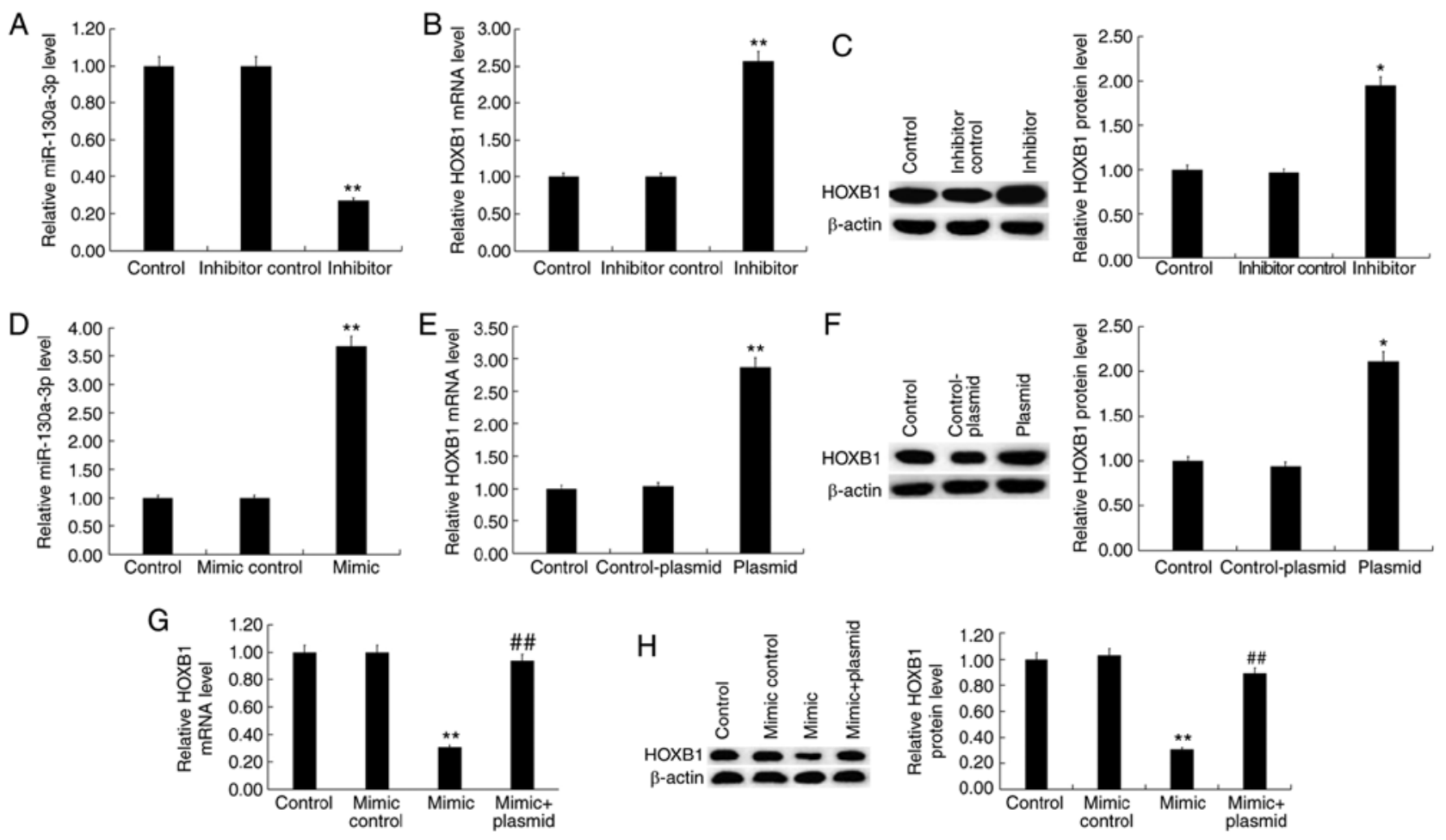

Figure 4. Expression of miR-130a-3p and HOXB1 in Jurkat T cells. (A and D) RT-qPCR was used to detect the expression of miR-130a-3p in Jurkat T cells that were transfected with inhibitor control, miR-130a-3p inhibitor, mimic control, or miR-130a-3p mimic for $48 \mathrm{~h}$. (B and C) RT-qPCR and western blotting were used to detect the mRNA and protein expression of HOXB1 in Jurkat T cells that were transfected with inhibitor control, or miR-130a-3p inhibitor for $48 \mathrm{~h}$. (E and F) RT-qPCR and western blotting were used to detect the mRNA and protein expression of HOXB1 in Jurkat T cells that were transfected with control-plasmid or HOXB1-plasmid for $48 \mathrm{~h}$. (G and H) RT-qPCR and western blotting were used to detect the mRNA and protein expression of HOXB1 in Jurkat $\mathrm{T}$ cells that were transfected with mimic control, miR-130a-3p mimic, or miR-130a-3p mimic+HOXB1-plasimd for $48 \mathrm{~h}$. Data are displayed as the mean \pm SD. ${ }^{*} \mathrm{P}<0.05$ and ${ }^{* *} \mathrm{P}<0.01$ vs. the control group; ${ }^{\# \#} \mathrm{P}<0.01$ vs. the mimic group. $\mathrm{SD}$, standard deviation.

Expression level of miR-130a-3p and HOXB1 in Jurkat T cells after transfection. To determine whether miR-130a-3p influenced $\mathrm{T}$ cell proliferation and apoptosis, Jurkat $\mathrm{T}$ cells were transfected with inhibitor control, miR-130a-3p inhibitor, mimic control, miR-130a-3p mimic, control-plasmid, HOXB1-plasmid, or miR-130a-3p mimic+HOXB1-plasmid for $48 \mathrm{~h}$. RT-qPCR or and western blot assays were performed to detect transfection efficiency of the mimic or plasmid. RT-qPCR results revealed that miR-130a-3p inhibitor significantly decreased the expression of miR-130a-3p in Jurkat $\mathrm{T}$ cells, and it significantly upregulated the mRNA and protein levels of HOXB1 (Fig. 4A-C). In addition, miR-130a-3p mimic significantly increased the expression of miR-130a-3p in Jurkat T cells, and it decreased the mRNA and protein levels of HOXB1 (Fig. 4D, G and H). RT-qPCR and western blot analyses also indicated that HOXB1-plasmid significantly improved the expression level of HOXB1 in Jurkat T cells (Fig. 4E and F).

Effect of miR-130a-3p on proliferation and apoptosis of Jurkat $T$ cells. In order to shed light on the function of miR-130a-3p in $\mathrm{T}$ cells, the effect of miR-130a-3p on the proliferation of Jurkat $T$ cells was first investigated. CCK- 8 assay results revealed that compared with the control group, miR-130a-3p 

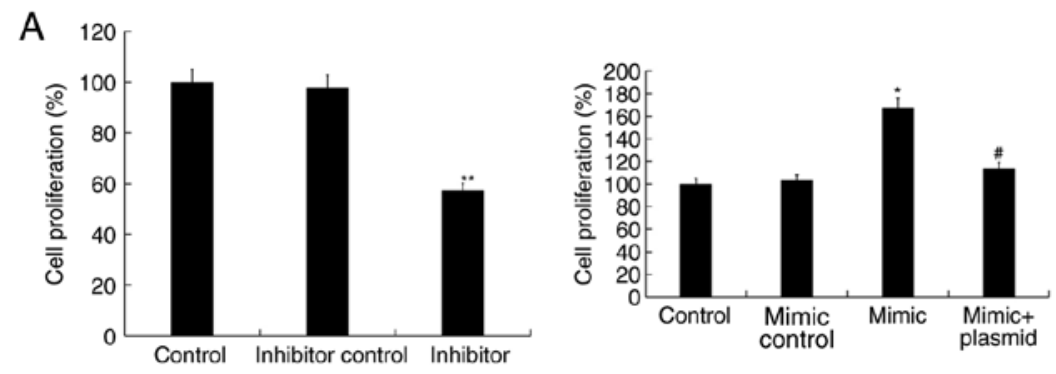

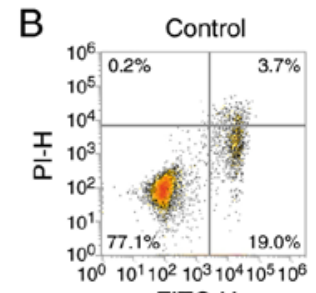

FITC-H

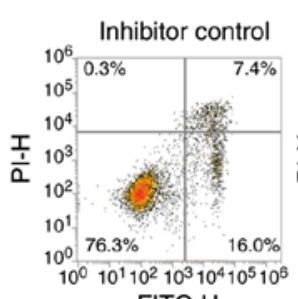

FITC-H

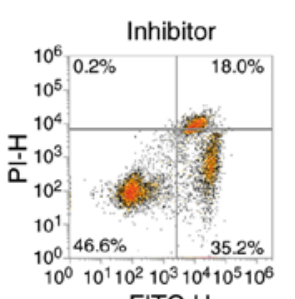

FITC-H

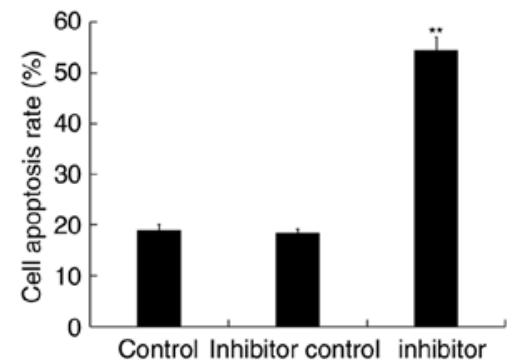

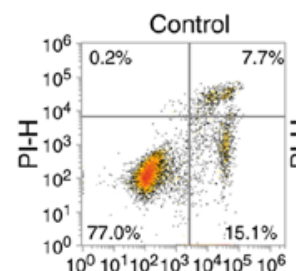

FITC-H

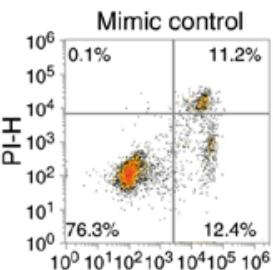

FITC-H

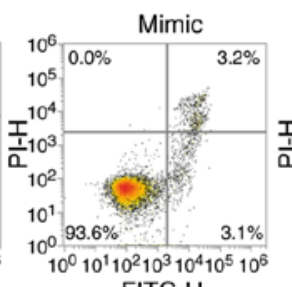

FITC-H

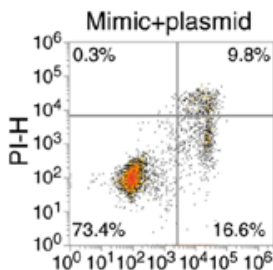

FITC-H

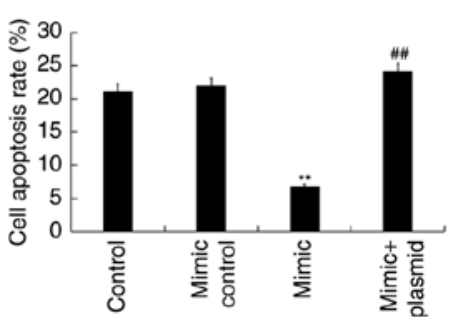

Figure 5. Effect of miR-130a-3p on cell proliferation and cell apoptosis of Jurkat T cells. (A) A CCK-8 assay was used to detect cell proliferation ability $48 \mathrm{~h}$ after Jurkat $\mathrm{T}$ cells were transfected with inhibitor control, miR-130a-3p inhibitor, mimic control, miR-130a-3p mimic, or miR-130a-3p mimic+HOXB1-plasmid. (B) A flow cytometric assay was used to detect cell apoptosis $48 \mathrm{~h}$ after Jurkat $\mathrm{T}$ cells were transfected with inhibitor control, miR-130a-3p inhibitor, mimic control, miR-130a-3p mimic, or miR-130a-3p mimic +HOXB1-plasmid. Data are displayed as the mean $\pm \mathrm{SD}$. ${ }^{*} \mathrm{P}<0.05$ and ${ }^{* *} \mathrm{P}<0.01$ vs. the control group; ${ }^{\#} \mathrm{P}<0.05$ and ${ }^{\# \#} \mathrm{P}<0.01$ vs. the mimic group. CCK-8, Cell Counting Kit-8; SD, standard deviation.
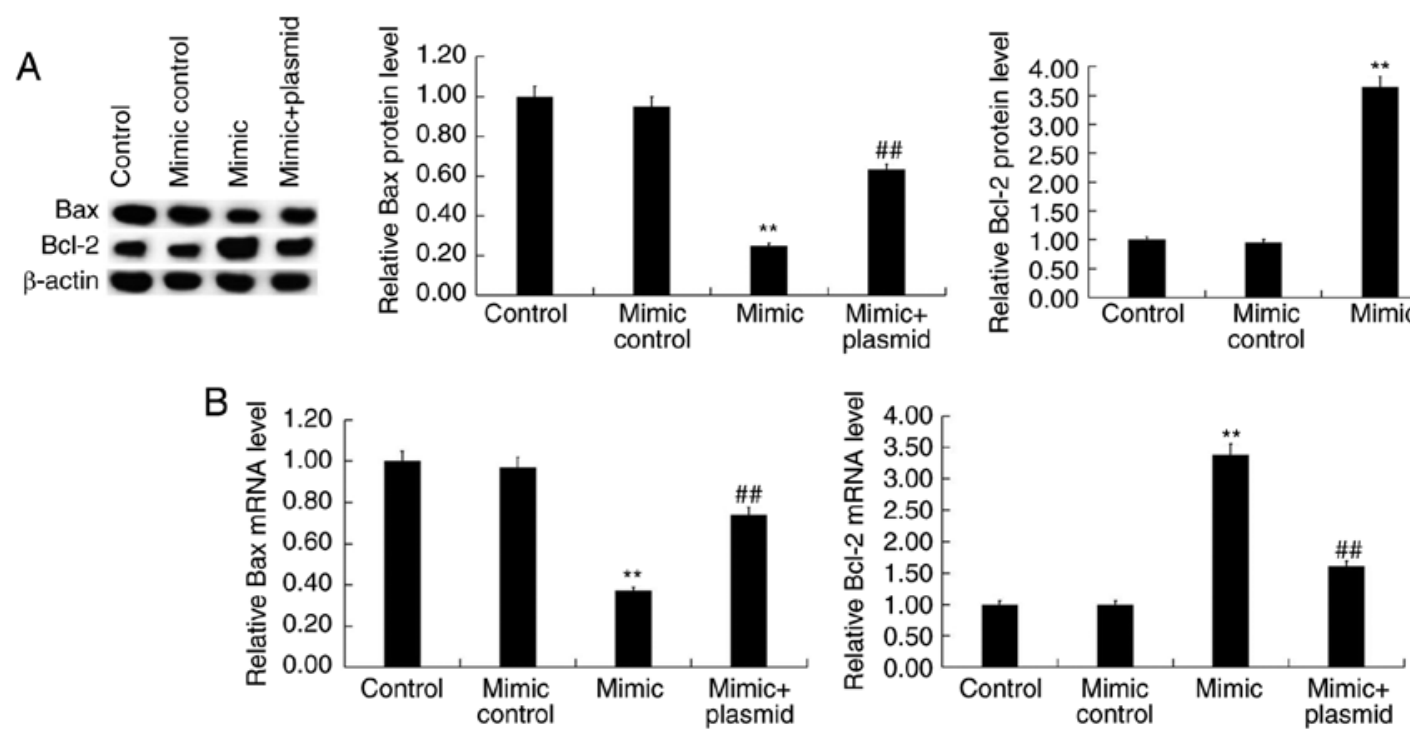

Figure 6. Effect of miR-130a-3p on Bax and Bcl-2 expression in Jurkat T cells. Forty-eight hours after Jurkat T cells were transfected with mimic control, miR-130a-3p mimic, or miR-130a-3p mimic+HOXB1-plasmid, (A) western blot assay and (B) RT-qPCR were performed to detect the protein and mRNA expression of Bax and Bcl-2 in Jurkat T cells. Data are displayed as the mean $\pm \mathrm{SD}$. ${ }^{* *} \mathrm{P}<0.01$ vs. the control group; ${ }^{\# \#} \mathrm{P}<0.01$ vs. the mimic group. Bcl-2, B-cell lymphoma 2; SD, standard deviation.

inhibitor significantly inhibited the cell proliferation activity of Jurkat T cells, while miR-130a-3p mimic significantly improved cell proliferation activity, and this increase was reversed by HOXB1-plasmid (Fig. 5A). To further determine whether miR-130a-3p regulated apoptosis, flow cytometry was performed to detect cell apoptosis. Flow cytometric analysis 
revealed that miR-130a-3p inhibitor significantly induced the cell apoptosis of Jurkat T cells, while miR-130a-3p mimic significantly decreased apoptosis, and this decrease was reversed by HOXB1-plasmid (Fig. 5B).

In addition, western blot analysis and RT-qPCR revealed that miR-130a-3p mimic significantly decreased Bax expression and increased B-cell lymphoma $2(\mathrm{Bcl}-2)$ expression at both the protein (Fig. 6A) and mRNA (Fig. 6B) level, which were reversed by HOXB1-plasmid.

\section{Discussion}

It has been confirmed that the pathogenesis of ankylosing spondylitis (AS) is closely related to HLA-B27. Furthermore, AS is a genetically heritable disease. There is increasing research suggesting the involvement of T cells in AS $(21,22)$. At present, there is still no cure method for AS treatment, thus it is urgent to find an effective target to cure AS clinically.

Wang et al demonstrated that miR-199a-5p could inhibit the pathogenesis of AS by targeting Ras homolog enriched in brain (Rheb) (23). In addition, miR-199a-5p was expressed at a low level in T cells from AS patients and could induce autophagy of $\mathrm{T}$ cells. Hou et al indicated that miR-let-7i could induce cell autophagy to protect $\mathrm{T}$ cells from apoptosis by targeting IGF1R (24). In the present study, it was revealed that miR-130a-3p was downregulated in $\mathrm{T}$ cells in HLA-B27-positive patients compared with the healthy controls.

In the study, it was determined that HOXB1 was a direct target gene of miR-130a-3p, and HOXB1 was upregulated in AS. Next, to investigate the relationship between miR-130a-3p and HOXB1. Jurkat $\mathrm{T}$ cells were transfected with inhibitor control, miR-130a-3p inhibitor, mimic control, miR-130a-3p mimic, or miR-130a-3p mimic+HOXB1-plasmid. The results revealed that miR-130a-3p negatively regulated the expression of HOXB1 in Jurkat T cells.

Numerous $\mathrm{T}$ cells have been revealed to be required for immune response in AS (25). There are increasing findings suggesting the involvement of T cells in AS (17,19). Recent studies revealed that disordered $\mathrm{T}$ cells were observed in peripheral blood and inflammatory joints from patients with AS $(26,27)$. A previous study indicated that let-7i played an important role in AS development by controlling T-cell fates by targeting IGF1R (22). In the present study, it was thus determined whether miR-130a-3p could affect T-cells fates, and cell proliferation and cell apoptosis was then analyzed. The findings of our present study indicated that miR-130a-3p downregulation inhibited cell proliferation ability and induced cell apoptosis of Jurkat T cells, while miR-130a-3p overexpression significantly promoted cell proliferation and inhibited cell apoptosis. The B-cell lymphoma 2 gene (Bcl-2) is a member of a family of proteins whose major function is their involvement in the initiation phase of the intrinsic pathways of apoptosis $(28,29)$. Bcl-2 has been demonstrated to be associated with apoptosis (30). Bcl-2-like protein 4 ( $\mathrm{Bax}$ ) is a pro-apoptotic protein of the Bcl-2 family of proteins (31). However, $\mathrm{HOXB1}$ has been reported to regulate the $\mathrm{Bax} / \mathrm{Bcl}-2$ ratio thus participating in the regulation of cell apoptosis (32). The effect of miR-130a-3p on Bcl-2 and Bax expression was then studied in Jurkat T cells. The results revealed that miR-130a-3p mimic significantly reduced Bax expression and increased Bcl-2 expression at both the protein and mRNA levels. It is worth mentioning that all the effects of miR-130a-3p mimic on Jurkat $\mathrm{T}$ cells were eliminated by HOXB1 overexpression. In recent years, miRNA target protectors have been investigated for more in-depth research of the physiological roles of specific miRNA-mRNA pairs $(33,34)$. In the present study, the HOXB1 plasmid was used to increase the expression of HOXB1, which was decreased by the miR-130a-3p mimic. Whether miRNA target protectors can specifically block the inhibition of HOXB1 by miR130a-3p requires further investigation.

In summary, our data revealed that miR-130a-3p was decreased in T cells of HLA-B27-positive AS patients. Furthermore, it was revealed that HOXB1 was a direct target gene of miR-130a-3p, and HOXB1 was upregulated in T cells of HLA-B27-positive AS patients. In addition, our data suggested that miR-130a-3p overexpression could improve Jurkat $\mathrm{T}$ cell proliferation and inhibit cell apoptosis, which were reversed by HOXB1-plasmid. Collectively, the present study suggested that miR-130a-3p may affect T-cell fate in AS, and thus participate in AS development. However, this is a preliminary study of the role of miR-130a-3p in AS. In addition, there were some limitations in the present study: i) The expression of miR-130a-3p in HLA-B27-positive patients and HLA-B-27-negative patients with was not compared; the role of HOXB1 in T-cell survival was not investigated. To ascertain the role of miR-130a-3p in AS, more research is still required. For example, the expression of miR-130a-3p in HLA-B27-negative AS patients and HLA-B27 positive healthy donors should be determined. The role of HOXB1 alone in T-cell survival should also be addressed. The underlying mechanism of how T-cell reduction contributes to AS development requires further study. In the future, these issues will be addressed.

\section{Acknowledgements}

Not applicable.

\section{Funding}

No funding was received.

\section{Availability of data and materials}

The data sets used during the present study are available from the corresponding author upon reasonable request.

\section{Authors' contributions}

FL designed the study, drafted the manuscript and performed literature search. FL, DS and XG collected the data and performed statistical analysis. NG, DL, LZ, XJ and JM performed statistical analysis and interpreted the data. All authors read and approved the final manuscript.

\section{Ethics approval and consent to participate}

The present study was approved by The Ethics Committee of Puyang Oilfield General Hospital (Puyang, China) and written informed consent was obtained from each patient. 


\section{Patient consent for publication}

Not applicable.

\section{Competing interests}

The authors state that they have no competing interests.

\section{References}

1. Dougados M and Baeten D: Sphodyloarthritis. Lancet 377: 2127-2137, 2011

2. Evans DM, Spencer CC, Pointon JJ, Su Z, Harvey D, Kochan G, Oppermann U, Dilthey A, Pirinen M, Stone MA, et al: Interaction between ERAP1 and HLA-B27 in ankylosing spondylitis implicates peptide handling in the mechanism for HLA-B27 in disease susceptibility. Nat Genet 43: 761-767, 2011.

3. Hou ZD, Xiao ZY, Gong Y, Zhang YP and Zeng QY: Arylamine $\mathrm{N}$-acetyltransferase polymorphisms in Han Chinese patients with ankylosing spondylitis and their correlation to the adverse drug reactions to sulfasalazine. BMC Pharmacol Toxicol 15: 64-80, 2014.

4. Liu Z, Zhang P and Dong J: Genetic variants of STAT4 are associated with ankylosing spondylitis susceptibility and severity in a Chinese Han population. Int J Clin Exp Med 7: 5877-5881, 2014.

5. Dean LE, Jones GT, MacDonald AG, Downham C, Sturrock RD and Macfarlane GJ: Global prevalence of ankylosing spondylitis. Rheumatology (Oxford) 53: 650-657, 2014.

6. Haywood KL, Packham JC and Jordan KP: Assessing fatigue in ankylosing spondylitis: The importance of frequency and severity. Rheumatology 53: 552-556, 2014.

7. Gan FY, Fei YY, Li MT, Wang Q, Xu D, Hou Y, Zeng XF and Zhang FC: The characteristics of patients having ankylosing spondylitis associated with Takayasu's arteritis. Clin Rheumatol 33: 355-358, 2014.

8. Li X, Liu F, Lin B, Luo H, Liu M, Wu J, Li C, Li R, Zhang X, Zhou K and Ren D: miR-150 inhibits proliferation and tumorigenicity via retarding G1/S phase transition in nasopharyngeal carcinoma. Int J Oncol 50: 1097-1108, 2017.

9. Ro S, Park C, Young D, Sanders KM and Yan W: Tissue-dependent paired expression of miRNAs. Nucleic Acids Res 35: 5944-5953 2007.

10. Mallory AC and Vaucheret H: MicroRNAs: Something important between the genes. Curr Opin Plant Biol 7: 120-125, 2004

11. Garzon R, Calin GA and Croce CM: MicroRNAs in cancer. Annu Rev Med 60: 167-179, 2009.

12. Gaur N,Karouzakis E, Gluck S, Bagdonas E, Jüngel A, Michel BA, Gay RE, Gay S, Frank-Bertoncelj M and Neidhart M: Micrornas interfere with DNA methylation in rheumatoid arthritis synovial fibroblasts. RMD Open 2: e000299, 2016.

13. Freiesleben S, Hecker M, Zettl UK, Fuellen G and Taher L: Analysis of microRNA and gene expression profiles in multiple sclerosis: Integrating interaction data to uncover regulatory mechanisms. Sci Rep 6: 34512, 2016.

14. Lai NS, Yu HC, Chen HC, Yu CL, Huang HB and Lu MC: Aberrant expression of microRNAs in T cells from patients with ankylosing spondylitis contributes to the immunopathogenesis. Clin Exp Immunol 173: 47-57, 2013.

15. Jiang Y and Wang L: Role of histone deacetylase 3 in ankylosing spondylitis via negative feedback loop with microRNA-130a and enhancement of tumor necrosis factor-1 $\alpha$ expression in peripheral blood mononuclear cells. Mol Med Rep 13: 35-40, 2016

16. Shah N and Sukumar S: The Hox genes and their roles in oncogenesis. Nat Rev Cancer 10: 361-371, 2010.
17. Han L, Liu D, Li Z, Tian N, Han Z, Wang G, Fu Y, Guo Z, Zhu Z Du C and Tian Y: HOXB1 Is a Tumor Suppressor Gene Regulated by miR-3175 in Glioma. PLoS One 10: e0142387, 2015.

18. Livak KJ and Schmittgen TD: Analysis of relative gene expression data using real-time quantitative PCR and the (-Delta Delta C(T)) method. Methods 25: 402-408, 2001.

19. Su JR, Kuai JH and Li YQ: Smoc2 potentiates proliferation of hepatocellular carcinoma cells via promotion of cellcycle progression. World J Gastroenterol 22: 10053-10063, 2016.

20. Jiang Y, Wang W, Liu ZY, Xie Y, Qian Y and Cai XN Overexpression of miR-130a-3p/301a-3p attenuates high glucoseinduced MPC5podocyte dysfunction through suppression of TNF- $\alpha$ signaling. Exp Ther Med 15: 1021-1028, 2018.

21. Appel H, Maier R and Bleil J, Hempfing A, Loddenkemper C, Schlichting U, Syrbe U and Sieper J: In situ analysis of interleukin-23-and interleukin-12-positive cells in the spine of patients with ankylosing spondylitis. Arthritis Rheum 65: 1522-1529, 2013.

22. Syrbe U, Scheer R, Wu P and Sieper J: Differential synovial Th1 cell reactivity towards Escherichia coli antigens in patients with ankylosing spondylitis and rheumatoid arthritis. Ann Rheum Dis 71: 1573-1576, 2012.

23. Wang Y, Luo J, Wang X, Yang B and Cui L: MicroRNA-199a-5p induced autophagy and inhibits the pathogenesis of ankylosing spondylitis by modulating the mTOR signaling via directly targeting ras homolog enriched in brain (Rheb). Cell Physiol Biochem 42: 2481, 2017.

24. Hou C, Zhu M, Sun M and Lin Y: MicroRNA let-7i induced autophagy to protect $\mathrm{T}$ cell from apoptosis by targeting IGF1R. Biochem Biophys Res Commun 453: 728-734, 2014.

25. Zou J, Appel H, Rudwaleit M, Thiel A and Sieper J: Analysis of the CD8+ T cell response to the G1 domain of aggrecan in ankylosing spondylitis. Ann Rheum Dis 64: 722-729, 2005.

26. Wu Y, Ren M, Yang R, Liang X, Ma Y, Tang Y, Huang L, Ye J, Chen K, Wang P and Shen H: Reduced immunomodulation potential of bone marrow-derived mesenchymal stem cells induced CCR4+CCR6+ Th/Treg cell subset imbalance in ankylosing spondylitis. Arthritis Res Ther 13: R29, 2011.

27. Nistala K, Moncrieffe H, Newton KR, Varsani H, Hunter $P$ and Wedderburn LR: Interleukin-17-producing T cells are enriched in the joints of children with arthritis, but have a reciprocal relationship to regulatory T cell numbers. Arthritis Rheum 58: 875-887, 2008

28. Reed JC: Bcl-2 and the regulation of programmed cell death. J Cell Biol 124: 1-6, 1994

29. Hatok J and Racay P: Bcl-2 family proteins: Master regulators of cell survival. Biomol Concepts 7: 259-270, 2016

30. Thomas S, Quinn BA, Das SK, Dash R, Emdad L, Dasgupta S, Wang XY, Dent P, Reed JC, Pellecchia M, et al: Targeting the Bcl-2 family for cancer therapy. Expert Opin Ther Targets 17: $61-75,2013$

31. Gross A, Jockel J, Wei MC and Korsmeyer SJ: Enforced dimerization of $\mathrm{BAX}$ results in its translocation, mitochondrial dysfunction and apoptosis. EMBO J 17: 3878-3885, 1998.

32. Petrini M, Felicetti F, Bottero L, Errico MC, Morsilli O, Boe A, De Feo A and Carè A: HOXB1 restored expression promotes apoptosis and differentiation in the HL60 leukemic cell line. Cancer Cell Int 13: 101, 2013.

33. Staton AA and Giraldez AJ: Use of target protector morpholinos to analyze the physiological roles of specific miRNA-mRNA pairs in vivo. Nat Protoc 6: 2035-2049, 2011.

34. Knauss JL, Bian S and Sun T: Plasmid-based target protectors allow specific blockade of miRNA silencing activity in mammalian developmental systems. Front Cell Neurosci 7: 163, 2013. 